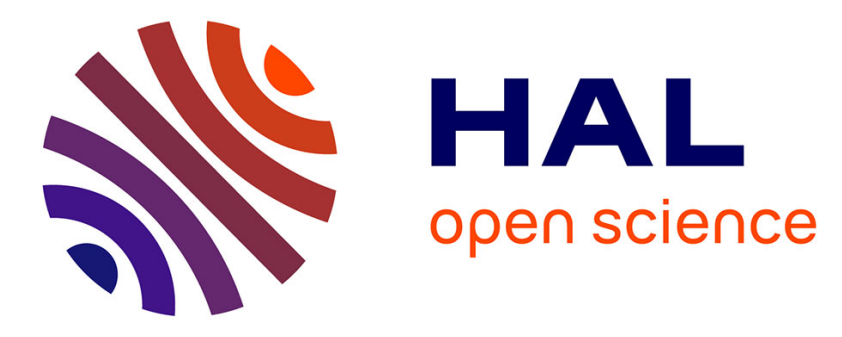

\title{
Design of Plant Eco-physiology Monitoring System Based on Embedded Technology
} Yunbing Li, Cheng Wang, Xiaojun Qiao, Yanfei Liu, Xinlu Zhang

\section{To cite this version:}

Yunbing Li, Cheng Wang, Xiaojun Qiao, Yanfei Liu, Xinlu Zhang. Design of Plant Eco-physiology Monitoring System Based on Embedded Technology. Third IFIP TC 12 International Conference on Computer and Computing Technologies in Agriculture III (CCTA), Oct 2009, Beijing, China. pp.185-190, 10.1007/978-3-642-12220-0_28 . hal-01061730

\section{HAL Id: hal-01061730 https://hal.inria.fr/hal-01061730}

Submitted on 8 Sep 2014

HAL is a multi-disciplinary open access archive for the deposit and dissemination of scientific research documents, whether they are published or not. The documents may come from teaching and research institutions in France or abroad, or from public or private research centers.
L'archive ouverte pluridisciplinaire HAL, est destinée au dépôt et à la diffusion de documents scientifiques de niveau recherche, publiés ou non, émanant des établissements d'enseignement et de recherche français ou étrangers, des laboratoires publics ou privés.

\section{(c)(1)}

Distributed under a Creative Commons Attribution| 4.0 International License 


\title{
DESIGN OF PLANT ECO-PHYSIOLOGY MONITORING SYSTEM BASED ON EMBEDDED TECHNOLOGY
}

\author{
Yunbing $\mathrm{Li}^{1,2}$, Cheng Wang ${ }^{1, *}$, Xiaojun Qiao ${ }^{1}$, Yanfei Liu ${ }^{1}$, Xinlu Zhang ${ }^{1}$ \\ 1 National Engineering Research Center for Information Technology in Agriculture, Beijing, \\ P. R. China 100097. \\ 2 Engineering college, South China Agricultural University, Guangzhou, Guangdong \\ Province, P.R. China. 510642. \\ *Corresponding Author adress: +86-010-51503409, Email:wangc@nercita.org.cn
}

Abstract: A real time system has been developed to collect plant's growth information comprehensively. Plant eco-physiological signals can be collected and analyzed effectively. The system adopted embedded technology: wireless sensors network collect the eco-physiological information. Touch screen and ARM microprocessor make the system work independently without PC. The system is versatile and all parameters can be set by the touch screen. Sensors' intelligent compensation can be realized in this system. Information can be displayed by either graphically or in table mode. The ARM microprocessor provides the interface to connect with the internet, so the system support remote monitoring and controlling. The system has advantages of friendly interface, flexible construction and extension. It's a good tool for plant's management.

Keywords: plant eco-physiology monitor system, embedded technology, MODBUS Protocol

\section{INTRODUCTION}

Plant eco-physiology is the research of interaction between plant and the environment. Physiological parameters included such as stem sap flow, stem diameter variation, transpiration and leaf temperature, plant ecological parameters include the ambient environment of plant such as the total irradiances, density of $\mathrm{CO} 2$, temperature and moisture of soil and so on. Plant eco-physiology signal can be monitored roundly and automatically by the system. Physiological parameters provide important information, especially if integrated into control systems or computer 
models in the so-called SPA (speaking plant approach) (Hashimoto et al., 1989). Lots of researches have showed that physiological parameters reflect the status of the plant growth and physiology demand directly and immediately (Meng Zhaojiang et al., 2005). The conventional routes of monitoring environmental parameters in greenhouse realize environment regulation achieve plant growth environment optimization (Yang Weizbong et al., 2006; Teng Guanghui et al.,2002). But plant growth's influence factors are complex, different environment are required for different plant, depending upon the environment parameters purely cannot applied to many plant species widely(S.L. Speetjens et al., 2008). The continuous and automatic monitoring system for plant ecophysiology is able to feed back the information of plant needing, and it is used for environment regulation when mismatches appear between environmental status and requirements of plant. In developed countries, plant physiological parameters monitored prevail in some orchards (Tom Helmer et al., 2008). But its apparatus communication limited in a small area, and its extension was inflexible. What's more, it needs the computer to support work. But these apparatus have similar shortcomings: functional simple, poor scalability, and the need for PC support. With the development of embedded technology, people use microprocessor as a substitute for computer. In this paper embedded technology and wireless sensor network were adopted to design plant eco-physiology monitoring system. The wireless sensing network collect information for ARM microprocessor, and the microprocessor realized the whole system's control and predicts status of plant from the collected information. The plant eco-physiology monitor system can be adopted in plant's management, water management and environment monitoring, which has forecast and guide functions.

\section{SYSTEM FRAMEWORK}

The system is organized as three parts: ARM controller module, data acquisition module and long-distance communication module. Wireless sensor network (WSN) with ZigBee function is utilized in the data acquisition module. The WSN gathers various plants' eco-physiological information and transmits it to the ARM controller. The controller, in which ARM micro-processor as its core and $\mu \mathrm{C} / \mathrm{OS}$-II real time operation system as its developing platform, it can control the whole system and make tasks scheduled. Touch screen is used as an output device which can display the information as well as input device. System parameters can be set according 
to user's needs. The monitor system communicates with server through Ethernet.

\section{SYSTEM'S HARDWARE DESIGN}

The ARM controller has many peripherals. The power source management module provides the multi-channel power sources for system models respectively. The WSN termination nodes connected with controller through RS232 interface. The Coordinator node of WSN exchange information with controller through ModBus protocol. Through the Internet system can exchange information with the long-distance server. People can browse information and realize the remote operation through the Internet.

\subsection{Resource of ARM controller}

The core of embedded system is based on ARM7TDMI processor. The processor connects with the programmable memory flash, data storage flash, Ethernet controller chip CS8900A, USB-Host controller. The ARM interface is shown in Fig. 1. It contains a flash on the board, a standard extend RTC real-time clock, UART controller, IIC and SPI Bus. The flash can storage 1 GB data, A real-time operating system $\mu \mathrm{C} / \mathrm{OS}-\mathrm{II}$ is used in this system, which supports the TCP/IP protocol and FAT document management. Resources of the system help us to realize the long-distance network access and control. The system supports the CF card for extended storage and Udisk makes information transmitted and saved conveniently.

\section{SOFTWARE DESIGN}

We can execute task scheduling and management by the $\mu \mathrm{C} / \mathrm{OS}-\mathrm{II}$ platform. $\mu \mathrm{C} / \mathrm{OS}-\mathrm{II}$ system supports 64 tasks at most. In this system we use 4 tasks respectively: Man-machine interface control, information collection and control, file management and peripheral device correspondences, remote access and control. Tasks priority dispatches according to time allocation in the main program. 


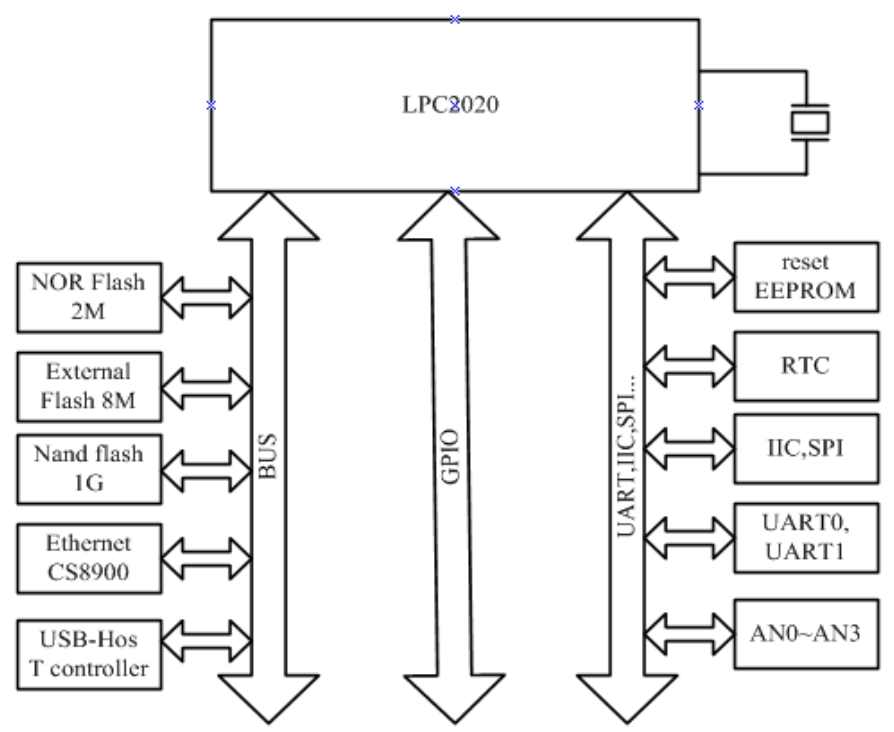

Fig.1. Interface of ARM

\subsection{The realization of GUI}

Touch screen in the design supports plenty of pictures save and graphic operation. Touch screen holds its own instruction set. All operations of the touch screen were implemented by sending a sequence of instructions in a certain format. In the project, we make the instruction set to sub-function for the convenience of calling. The sub-function format such as data source, operation set source, operator and operarand, the length of sub-function.

Func_Type Func_Name([*set] , [*source], [*operands], [length]);

Through calling the sub-function we can carry on the user interface operation such as text demonstrated, drawing and database operation. The main program call sub-function in GUI program realizes the system display control.

\subsection{System software}

The main program realizes the overall temperament of the system. After system powered on, program begin to work and the system realizes sensor auto-search in the area through the unique sensor ID. Compensation of sensor set parameters for polynomials, which standardizes the gathering data. Touch screen can display gathering information dynamically and may also review all history data. The information can also be displayed graphically. Watch-dog in software prevents system from halting. Back light of touch screen will be set to reduce the system's power loss. The primary tasks are 
shown in the Fig.2.

\subsubsection{The human-machine interaction interface}

The favorable man-machine interface and touch screen made the system operation simple. The controller has relative action according to different instructions. The system's human-machine interaction flow is shown as task 1 in Fig.2. The controller send commands to the LCD, then the LCD operating depends on commands parsing. And the controller distills the touch screen return information. The system can also display in multiple languages by download different word libraries. The touch screen can work as keyboard of PC.

\subsubsection{Communication between the controller and WSN}

MODBUS protocol was adopted to enhance system communication reliability between the controller and WSN. It makes different control devices in the industry network realization of system's centralized monitoring. There are two different transmission patterns: the ASCII pattern and the RTU pattern. RTU pattern is used in this design. The system maps to sensor through unique sensor ID, query WSN data transmission through MODBUS protocol. When the system measure time is up, the communication task was executed, the data in the buffer of collect node was sending to the controller. And in the system sensor set, all the parameter set by user was downloaded to the WSN. System communication program flow is shown as task 3 in Fig.2.

\subsubsection{Data processing}

In the software designing all sensors are defined as class. Relative operation of sensor depending on sensor's ID in program. Sensors attributes can be set by the user through the touch screen. Double-linked list is applied to carry on the dynamic node assignment for enhancing memory allocated. The operation of create or delete sensor node easily, and traversal all sensors which exists in the system very conveniently. Sensor information includes sensor's ID, sampling period, name and value. Compensation mathematical formula realized sensor intelligent compensation. After collecting data, system will transform it according to the offset parameter of the compensation formula. After the sensor loaded once, system will record it automatically, which will help sensor auto-search in the next time. All data acquired by the sensor is appended to the data saved document according sensors' ID. The corresponding time and the sensor value will be saved in file. All information will be saved in the board flash. It has 1GB capacity and able to export its information to $\mathrm{U}$ disk easily. All documents are saved 
in a certain format and exported to generate document of EXCEL format, which help data processing easily. The flow diagram is shown as task 2 in Fig.2.

\subsubsection{Remote monitoring and control}

Server and system are connected by the $\mathrm{B} / \mathrm{S}$ pattern. The communication is based on the TCP/IP network protocol. The controller listening in the port assign by server realize the server and the control system long-distance communication. All information frames transmit in network socket character. The system receive and action as the orders from the server. The flow diagram is shown as task 4 in Fig.2.

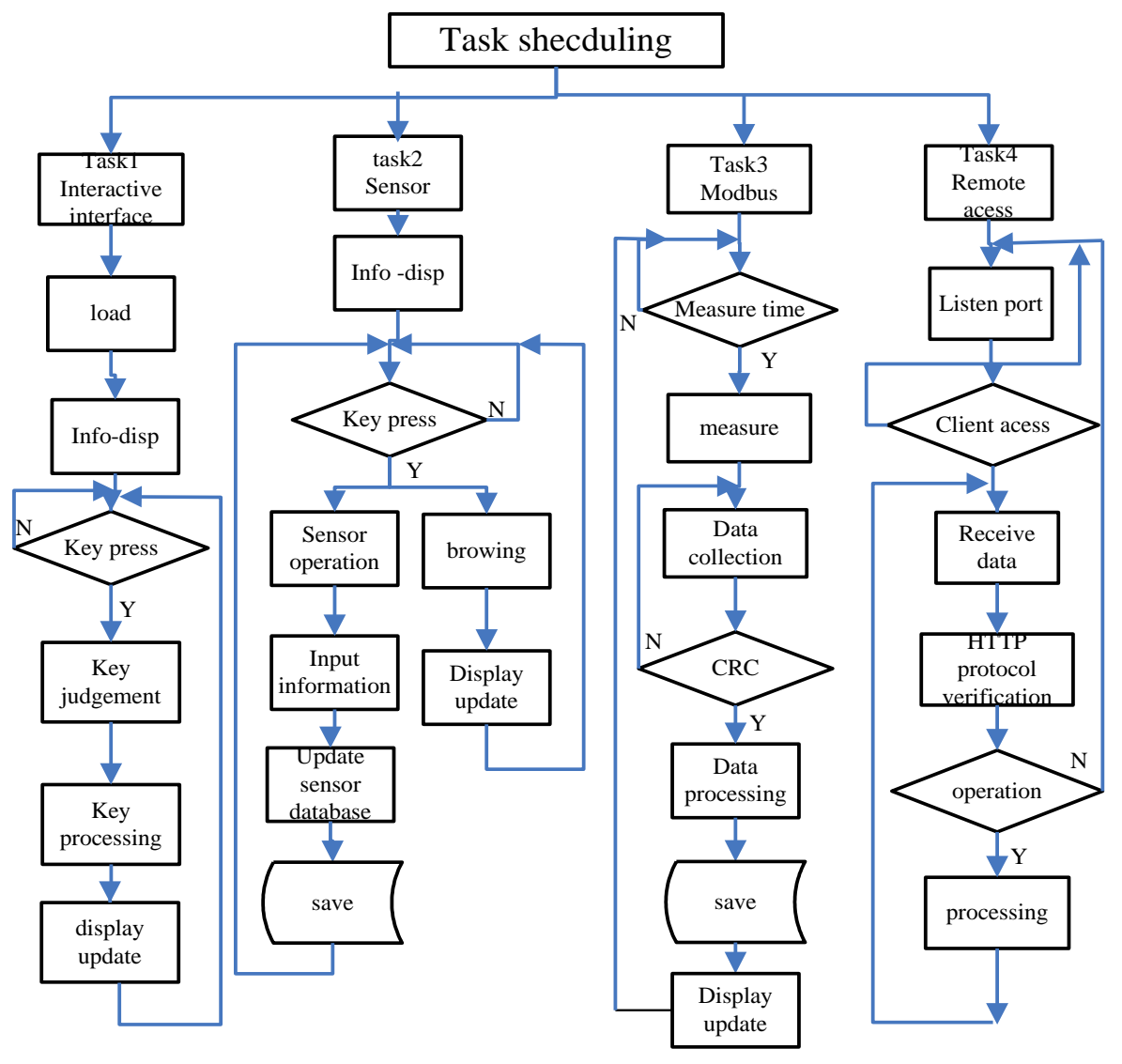

Fig.2. Main program scheme 


\section{DISCUSSION}

System meets the requirements of analysis and monitoring plant ecophysiology information, which will alarm automatically when the ecophysiology information becomes abnormal. The system has the following features, such as flexibility, reliability, simple operation and scalability. System can be used as an accessorial tool for greenhouse control, which can support decision for greenhouse intelligent management.

The next step of developing, "knowledge-base management system" will be added in this system. According to different flowers and crops ' growth rule, revise the system set points to achieve environment optimization for plant. Working out optimization environment modeling for plant based on monitor and analysis of the plant eco-physiological parameters. As a result, system also has functions of alarm plant stress of water deficit, pest or environmental stress. Forecast and tutor in water-saving irrigation, accurate control and so on. It helps people to manage plant scientifically and use the resources more reasonably.

\section{CONCLUSIONS}

We designed a plant eco-physiological monitoring system based on embedded technology. The following conclusions were inferred from the study:

1. Hardware platform of autonomous data collection system was developed based on ARM microprocessor. It connects with wireless sensor network and dynamic control of data collection was realized.

2. Touch screen was adopted, operation was very simply, and interface was friendly.

3. The system can work independent without PC. What's more, through internet it can connect with server to achieve remote monitoring.

\section{ACKNOWLEDGEMENTS}

This research was supported by the National High Technology R\&D Program (863 Program 2008AA10Z201), National Support Science and Technology Program (2006BAD11A10, 2006BAD30B03) and Beijing Municipal Science and Technology Program: Development and 
demonstration of voice-based wireless ad hoc network monitoring system in facilities environmental.

\section{REFERENCES}

Hashimoto, Y. . Recent strategies of optimal regulation by the speaking plant concept, Acta. Hortic, 1989, 260:115-121

S.L. Speetjens, H.J.J. Janssen, G. van Straten etal. Methodic design of a measurement and control system for climate control in horticulture, Computers and Electronics in Agriculture, 2008, 64:162-172

Tom Helmer, David L. Ehret, Shabtai Bittman. CropAssist, an automated system for direct measurement of greenhouse tomato growth and water use, Computers and Electronics in Agriculture, 2005, 48:198-215

Meng Zhaojiang, Duan Aiwang, Liu Zugui, et a1. Advances on diagnosis of crop moisture content from changes in stem diameters of plants, Transactions of the CSAE, 2005, 21(2):

30-33 (in Chinese)

Yang Weizbong, Wang Yiming, Li Haijian. Distributed greenhouse intelligent control system based on the field bus concept, Transactions of the CSAE, 2006, 22(9): 163-167(in Chinese)

Teng Guanghui, Li Changying. DNCS - A New Scheme for the Automation of Greenhouse Environment Control, Transactions of the CSAE, 2002, 18(5):118-122(in Chinese) 